\title{
Coulomb explosion of K-shell ionized krypton clusters studied by multiple-ion coincidence momentum imaging
}

\section{$\operatorname{AUTHOR}(S)$ :}

Iwayama, H; Nagaya, K; Murakami, H; Ohmasa, Y; Yao, M

\section{CITATION:}

Iwayama, $\mathrm{H}$...[et al]. Coulomb explosion of K-shell ionized krypton clusters studied by multiple-ion coincidence momentum imaging. JOURNAL OF CHEMICAL PHYSICS 2007, 126(2): 024305.

\section{ISSUE DATE:}

2007-01-14

URL:

http://hdl.handle.net/2433/49826

\section{RIGHT:}

Copyright 2007 American Institute of Physics. This article may be downloaded for personal use only. Any other use requires prior permission of the author and the American Institute of Physics. 


\title{
Coulomb explosion of $\boldsymbol{K}$-shell ionized krypton clusters studied by multiple-ion coincidence momentum imaging
}

\author{
H. Iwayama, K. Nagaya, ${ }^{a)}$ H. Murakami, Y. Ohmasa, and M. Yao \\ Department of Physics, Graduate School of Science, Kyoto University, Sakyo-ku, Kyoto 606-8502, Japan
}

(Received 12 September 2006; accepted 14 November 2006; published online 11 January 2007)

\begin{abstract}
The Coulomb explosion of $K$-shell ionized krypton clusters with an average size $\langle N\rangle$ of 160 has been studied by electron-multiple-ion-coincidence measurements in which the time-of-flight (TOF) of ions was measured by using a position sensitive detector. The authors have sorted the TOF spectra by the number of coincidence ion signals, $N_{\text {coin }}$, and found that singly charged fragment ions such as $\mathrm{Kr}^{+}, \mathrm{Kr}_{2}^{+}$, and $\mathrm{Kr}_{3}^{+}$are dominant for $N_{\text {coin }} \geqslant 2$, and that multiply charged ions are detected mainly for $N_{\text {coin }}=1$. The $N_{\text {coin }}$ dependence of the peak widths in the TOF spectra reveals that the average momentum of the $\mathrm{Kr}^{+}$ions increases with $N_{\text {coin }}$, while those of $\mathrm{Kr}_{2}^{+}$and $\mathrm{Kr}_{3}^{+}$decrease. These results have been more directly confirmed by the momentum imaging measurements. The authors propose that the heavier ions are produced in the central part of clusters where the Coulomb interactions from the surrounding ions are more effectively canceled out due to the higher symmetry. (C) 2007 American Institute of Physics. [DOI: 10.1063/1.2406076]
\end{abstract}

\section{INTRODUCTION}

The Coulomb explosion of clusters and molecules has been extensively studied and observed in many experiments such as irradiation of intense laser, ${ }^{1-4}$ collision with highly charged ions, ${ }^{5,6}$ etc. When clusters and molecules are multiply ionized, they become unstable due to Coulomb repulsive forces and eventually dissociate into many fragments by Coulomb explosion. The momentum and kinetic energy of these fragment ions give information on the charge distribution just before the Coulomb explosion. In femtosecond laser experiments, for example, the structure of triatomic molecules in intense laser fields was investigated by analyzing momenta of their three fragment ions produced in the Coulomb explosion. ${ }^{3}$

Utilization of x-ray as a trigger of Coulomb explosion has manifold advantages. First, the excitation of clusters is achieved in a simple way (i.e., absorption of single photon). Second, the excitation is well localized because the core hole is created within an atom. Third, the number of positive charges generated as a result of the relaxation of inner-shell vacancies (Auger decay ${ }^{7,8}$ ) can be controlled by varying the photon energy. For example, a $2 p$-excited argon loses two to three electrons on average through Auger decay, while a $1 s$-excited argon atom loses about five electrons. ${ }^{8}$ Hence, the cluster experiment with $\mathrm{x}$-ray may give more helpful information on the fragmentation processes.

Until now tremendous efforts have been devoted to core excitation of clusters and molecules. ${ }^{9-22}$ In particular, experiments of Ar clusters with soft x-ray have been performed intensively. It was reported that $2 p$-excited Ar clusters become multiply charged through Auger decay and the positive charges are transported from the photoabsorbed atom to its neighbors, leading to fragmentation due to the Coulomb re-

\footnotetext{
a) Author to whom correspondence should be addressed. Electronic mail: nagaya@scphys.kyoto-u.ac.jp
}

pulsive forces (e.g., $\mathrm{Ar}_{7}^{2+} \rightarrow \mathrm{Ar}^{+}+\mathrm{Ar}_{2}^{+}+4 \mathrm{Ar}$ ). ${ }^{9,11-14}$ The relative abundance of fragment ions depends on the cluster size. In the case of triply charged Ar cluster, singly charged monomer ion $\mathrm{Ar}^{+}$is the most abundant species for $\langle N\rangle=10$, while $\mathrm{Ar}_{2}^{+}$becomes dominant for $\langle N\rangle=175$, $^{9,15}$

Although a pioneering work was done by Rühl et al. ${ }^{18}$ for Ar cluster with $\langle N\rangle \sim 400, K$-shell spectroscopy of raregas clusters has been rather limited, because the absorption cross section of hard $\mathrm{x}$-ray is considerably small compared with soft $\mathrm{x}$ ray. For $\mathrm{Kr}$ clusters the first electron-ioncoincidence (EICO) measurements by utilizing hard $\mathrm{x}$ ray have been carried out by Nagaya et al. recently. ${ }^{23}$ They found that dominant ionic species stemming from small neutral clusters are highly charged monomer ions, while for medium size clusters singly charged monomer ions become the most abundant. Then the fraction of singly charged dimers and subsequently that of trimers increase with increasing cluster size. They have interpreted the characteristic size dependence of the relative abundance of fragment ions by assuming site-dependent decay processes: when the core hole is generated on the cluster surface, the proliferated holes due to the Auger decay are strongly localized within the x-ray absorbing atom, while the charges are separated to surrounding atoms when the $\mathrm{x}$-ray absorption takes place inside the cluster. Although this picture is promising because it is consistent with many other experimental observations, ${ }^{5,20-22}$ more detailed experimental information on the decay processes is required.

For this purpose, it is desirable to detect all the ions participating in the Coulomb explosion and measure their momentum, from which charge distribution before the Coulomb explosion can be extracted. Thus we have developed a multiple-ion coincidence momentum imaging apparatus ${ }^{3,17}$ and applies it to $\mathrm{Kr}$ clusters. In the present work, electron$n$-ion coincidence time-of-flight (TOF) spectra of $\mathrm{Kr}$ cluster are measured up to $n=8$ by using a position sensitive detec- 


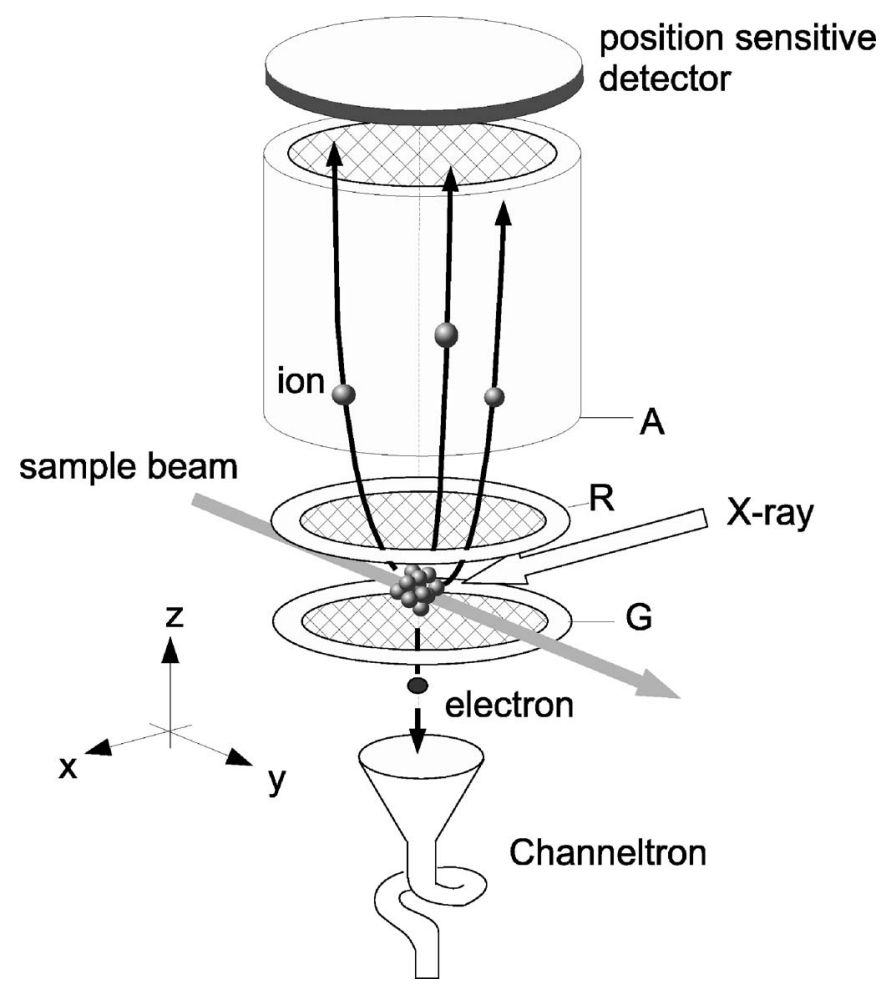

FIG. 1. A schematic view of the photoionization and detection parts of experimental apparatus where G, R, and A denote ground, repeller, and acceleration electrodes made of metallic mesh. The spacing between the ground and repeller electrode is $20 \mathrm{~mm}$. The voltages of the electrode, $U_{G}$, $U_{\text {Rep }}$, and $U_{\mathrm{Acc}}$, are $0,-400$, and $-1200 \mathrm{~V}$, respectively.

tor (PSD). The TOF spectra sorted by the number of coincidence ion signals is presented in Sec. III A of this paper. In Sec. III B detected positions on the PSD are presented, from which the momentum distributions of the fragment ions are deduced. In Sec. IV we discuss a mechanism of Coulomb explosion with special attention to the site-dependent decay processes.

\section{EXPERIMENT}

The electron- $n$-ion coincidence spectra were measured simultaneously with momentum imaging of ions for $\mathrm{Kr}$ atomic and cluster beams. The experiment was performed at the undulator beamline BL-37XU installed in SPring-8, where the high-brilliance $\mathrm{x}$-ray emitted from the undulator was monochromatized by a double crystal $\mathrm{Si}(111)$. The photon flux of $\mathrm{x}$-ray beam was about $4 \times 10^{12}$ photons/s during our measurements. The measurements were carried out at a photon energy of $14.50 \mathrm{keV}$, i.e., slightly higher than the $\mathrm{Kr}$ $K$ edge $(=14.327 \mathrm{keV})$.

Krypton clusters were produced by a supersonic expansion through a cylindrical nozzle $(\phi=60 \mu \mathrm{m})$. The stagnation pressure was 2 bar and the nozzle temperature was $150 \mathrm{~K}$, from which the average number of atoms within a cluster $\langle N\rangle$ was estimated to be $\sim 160$ by using Hagena's scaling law. ${ }^{10,24}$ Sample beams were shaped by a skimmer $(\phi=1 \mathrm{~mm})$. A schematic view of the photoionization and detection parts of the experimental apparatus is presented in

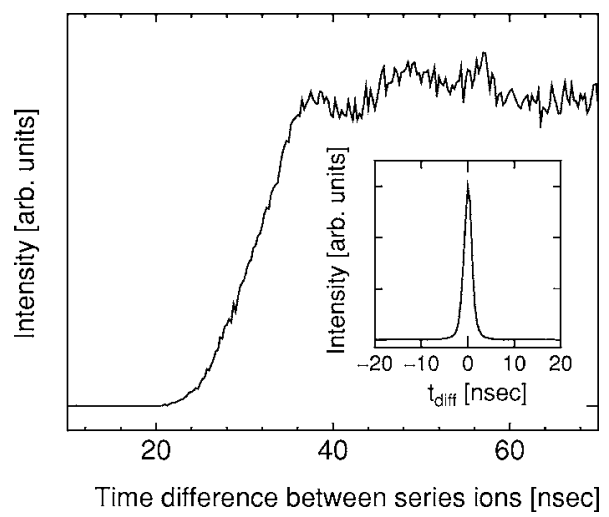

FIG. 2. Time difference between series ions (see Sec. IV). The leading edge of the plot corresponds to the electronic dead time (20 ns). The inset is the distribution of $t_{\text {diff }}=t_{x 1}+t_{x 2}-t_{y 1}-t_{y 2}$. The full width at half maximum of the peak is $1.9 \mathrm{~ns}$.

Fig. 1. The sample beam and the $\mathrm{x}$-ray beam intersected at a right angle in the horizontal plane, and the $\mathrm{x}$-ray polarization was parallel to the direction of the sample beam. Then electrons and ions were emitted from the sample beam through the absorption of an x-ray photon. The ions were deflected upward by a constant electric field to a modified WileyMcLaren-type time-of-flight mass spectrometer ${ }^{25}$ and detected by a PSD while the photoelectrons and Auger electrons were deflected downward and immediately $(<5 \mathrm{~ns})$ detected by a channeltron. The probability that neither photoelectron nor Auger electrons were detected on each photoabsorption was negligibly small. The electron detection signals were used as start signals for the time of flight, as in conventional EICO measurements.

For coincident detection of up to eight ions as well as their momenta, we used a multihit PSD system composed of a microchannel plate (MCP) and a delay-line anode detector (RoentDek DLD40). Signals from the detector were fed into a multistop time-to-digital converter (RoentDek TDC8). By using this system, the time of flight $T$ and the detection position $(X, Y)$ on PSD were recorded for each ion. The origin $(X, Y)=(0,0)$ was defined as the center of PSD. The full time range was $32 \mu \mathrm{s}$, and the active detector diameter of PSD was $47 \mathrm{~mm}$. The detection efficiency of the PSD was estimated to be $\sim 35 \%$ from the average number of detected ions per photoabsorption event for a krypton atomic beam. The efficiency was mainly determined by the active area of MCP. Further details of the experimental apparatus except for the detection part are described elsewhere. ${ }^{23}$

In the present experiments, we recorded four signals $\left(t_{x 1}, t_{x 2}, t_{y 1}, t_{y 2}\right)$ for each detected ion, where $t_{x 1}$ is the time required for the ion signal to travel along the delay line and reach the positive $x$ end of the detector, $t_{x 2}$ that to reach the negative $x$ end, $t_{y 1}$ that to reach the positive $y$ end, and $t_{y 2}$ that to reach the negative $y$ end. If some of four signals are lost due to the electronic dead time, we cannot obtain the detection position. We have removed these false coincidences from the raw data by the following two steps. First, we counted the number of these four signals for each photoabsorption event and rejected the event unless all the numbers of signals were the same. Second, we examined the time 
difference, $t_{\text {diff }}=t_{x 1}+t_{x 2}-t_{y 1}-t_{y 2}$, because it should be null for the true coincidences. The inset of Fig. 2 shows the distribution of $t_{\text {diff }}$. We accepted such signals that are included in the sharp peak centered at $t_{\text {diff }}=0$ as true coincidence.

\section{RESULTS}

\section{A. Time-of-flight mass spectra}

A TOF mass spectrum for a krypton atomic beam is represented in Fig. 3(a). The spectrum reveals that a krypton atom ionized by absorbing an $\mathrm{x}$-ray photon with $14.50 \mathrm{keV}$ loses many electrons through Auger decay and becomes multiply charged ions. The relative abundance of multiply charged ions $\mathrm{Kr}^{z+}(z \leqslant 11)$ is in good agreement with the previous result in the literature, ${ }^{8}$ and the average charge number is 6 . Note that singly charged atomic ions $\mathrm{Kr}^{+}$are hardly observed and doubly charged ions are also very few. Each peak in the TOF spectrum is so sharp that subpeaks due to many isotopes can be resolved in accordance with the natural abundance $\left(2 \%{ }^{80} \mathrm{Kr}, 11 \%{ }^{82} \mathrm{Kr}, 11 \%{ }^{83} \mathrm{Kr}, 56 \%{ }^{84} \mathrm{Kr}\right.$, $17 \%{ }^{86} \mathrm{Kr}$ ) from which the relative time resolution $T / \Delta T$ is estimated to be 220 .

A total TOF spectrum for the cluster beam $(\langle N\rangle \sim 160)$ is shown in Fig. 3(b), where the background spectrum is subtracted but the contribution from uncondensed atoms, whose atomic fraction was estimated to be $\sim 30 \%,{ }^{23}$ is included. Beside the multiply charged ions, new peaks corresponding to $\mathrm{Kr}_{n}^{z+}(n / z=1,2$, and 3) are clearly observed. Since the multiply charged rare-gas cluster ions are known to be unstable, unless the size of cluster ion is sufficiently large, ${ }^{26,27}$ these new peaks can be safely assigned to be singly charged ions $\mathrm{Kr}_{n}^{+}(n=1,2,3)$ (i.e., $\left.z=1\right)$. Compared with a single-stop TOF spectrum, ${ }^{23}$ the peaks due to $\mathrm{Kr}_{n}^{+}$are considerably enhanced in the multihit spectrum. The linewidth of singly charged ions is considerably broader than that of multiply charged ions, suggesting that the singly charged ions are products of Coulomb explosion. The base line between the peaks is structureless but has a finite value, indicating that accidental coincidences with false start triggers of TOF cannot be ignored in the present experiment. Thus the true coincidence TOF spectrum is deduced for each photodissociation event by eliminating those signals that can be assigned neither to the multiply charged atomic ions $\left(\mathrm{Kr}^{z+}(z \geqslant 2)\right)$ nor to the singly charged atomic or cluster ions $\left(\mathrm{Kr}_{n}^{+}\right.$ $(n=1,2,3))$ from raw multihit data. Then the coincidence spectra for various photodissociation events are sorted by the number of coincidence ion signals, $N_{\text {coin }}$, and summed up for each $N_{\text {coin }}$.

The coincidence TOF spectra summed up in this way are displayed in Fig. 3(c) for $N_{\text {coin }}=1-8$. Although the multiply charged ions $\mathrm{Kr}^{2+}$ are also generated from the cluster beam, they are abundant only for $N_{\text {coin }}=1$ and the width of their peaks remains very narrow. This indicates that $\mathrm{Kr}^{2+}$ are not products of Coulomb explosion even for the cluster beam. Moreover, the intensity distribution of $\mathrm{Kr}^{z+}$ peaks $(z \geqslant 2)$ for the cluster beam is nearly the same as that for the atomic beam, implying that it is not likely to occur that $\mathrm{Kr}^{2+}$ lose their charges partially to become another multiply charged ion $\mathrm{Kr}^{z^{\prime}+}$ with $z>z^{\prime}>1$.

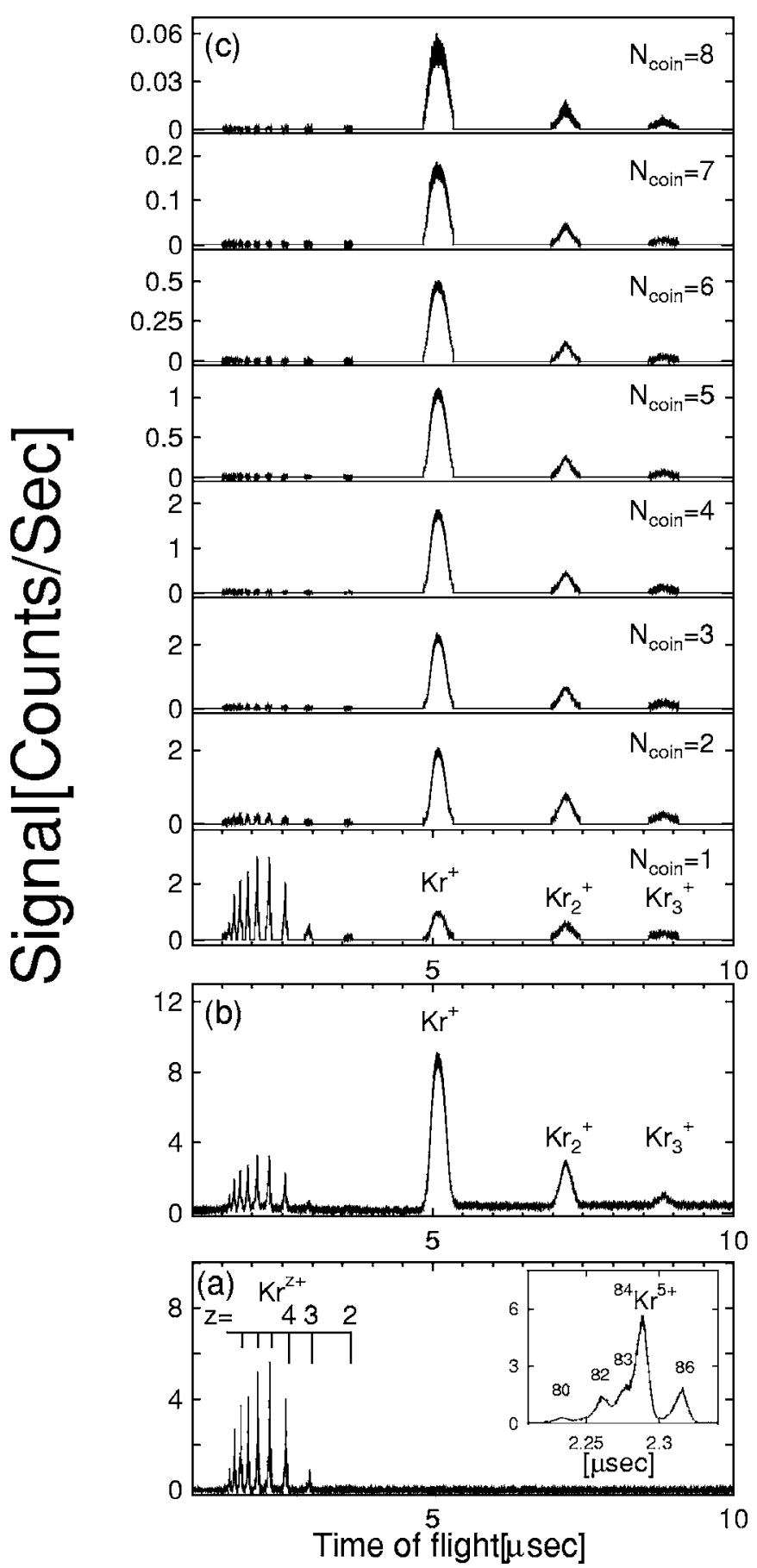

FIG. 3. Time-of-flight (TOF) mass spectra recorded with a photon energy of $14.50 \mathrm{keV}$. (a) TOF spectrum for a $\mathrm{Kr}$ atomic beam. The inset shows an expansion of the $\mathrm{Kr}^{5+}$ peak. (b) Multihit total TOF spectrum for a $\mathrm{Kr}$ cluster beam with $\langle N\rangle \sim 160$. (c) Multihit TOF spectra sorted by the number of coincidence ion signals $N_{\text {coin }}$ for the cluster beam.

For $N_{\text {coin }} \geqslant 2$, almost all ions are singly charged, indicating that the fragment ions generated by Coulomb explosion are predominantly singly charged. The relative abundance of $\mathrm{Kr}_{n}^{+}$is plotted for $n=1,2,3$ as a function of $N_{\text {coin }}$ in Fig. 4, where small contribution from multiply charged ions is ignored. The monomer ions $\mathrm{Kr}^{+}$are the most abundant and become more dominant as $N_{\text {coin }}$ increases. As seen from the vertical scale of Fig. 3(c), the event rate decreases rapidly with increasing $N_{\text {coin }}$, which is shown by the open circles in Fig. 5. 


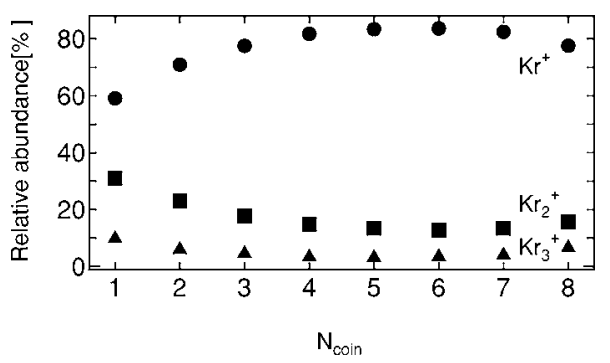

FIG. 4. Relative abundance of fragment ions $\mathrm{Kr}^{+}(\mathbf{\bullet}) \mathrm{Kr}_{2}^{+}(\boldsymbol{\square})$, and $\mathrm{Kr}_{3}^{+}(\mathbf{\Delta})$. The contribution of multiply charged ions $\mathrm{Kr}^{z+}(z \geqslant 2)$ is not included.

It is very interesting to see how the peak shape in the TOF spectrum changes with $N_{\text {coin }}$ [see Fig. 3(c)]. For $N_{\text {coin }}$ $=1$ both the $\mathrm{Kr}^{+}$peak and $\mathrm{Kr}_{2}^{+}$peak have a Gaussian-type shape, but with increasing $N_{\text {coin }}$ the former becomes a domelike shape, while the latter becomes a cusplike shape. In addition the $\mathrm{Kr}_{3}^{+}$peak also exhibits a similar $N_{\text {coin }}$ dependence to the $\mathrm{Kr}_{2}^{+}$peak. Since the peak width is proportional to the average velocity of fragment ions, this result suggests that their momentum distribution depends on $N_{\text {coin }}$. Further experimental information on this problem is presented in the next section.

\section{B. PSD images}

In the present experiment, several fragment ions are detected by the PSD in each photodissociation event and their detected positions $\left(x_{j}, y_{j}\right)$ on the PSD are recorded. As in the case of the coincidence TOF spectrum, the true coincidence position data, $\left(x_{j}, y_{j}\right)$ for $j=1, \ldots, N_{\text {coin }}$, are derived from raw data by eliminating accidental coincidence signals. Then the position data for various photodissociation events are sorted by $N_{\text {coin }}$ and also by the species of fragment ions, and finally the sorted data are compiled as PSD images.

The contour map in Fig. 6(a) shows a PSD image for a $\mathrm{Kr}$ atomic beam, where all the multiply charged ions are included. The contours are drawn every $20 \%$ of the maximum count. For the atomic beam, the PSD image exhibits a narrow peak whose center is slightly shifted toward the positive $Y$ direction because the atomic beam has a finite velocity. The full width at half maximum of the peak along the $X$ axis, $\Delta x$, is $6.2 \mathrm{~mm}$, and that along $Y$ axis, $\Delta y$, is $2.2 \mathrm{~mm}$. In contrast, singly charged ions from clusters have broad distributions [see Fig. 6(b)], which directly suggests that these ions are products of the Coulomb explosion. In particular, the distribution of $\mathrm{Kr}^{+}$is much broader than those of $\mathrm{Kr}_{2}^{+}$and

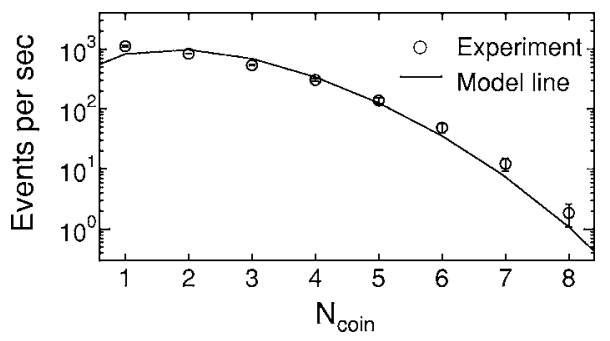

FIG. 5. Event rates for $N_{\text {coin }}=1-8$. Experimental data are denoted by circles with error bars, and a model calculation described in Sec. IV is shown by the curve. (a)

(c)
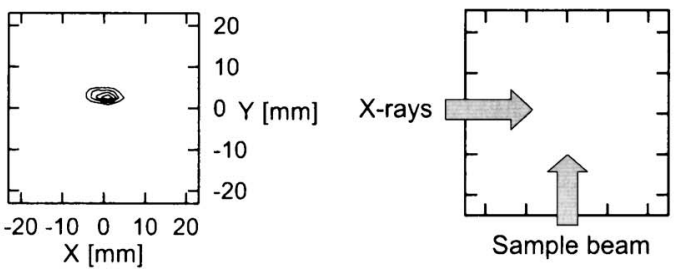

(b)

$$
\mathrm{Kr}^{+}
$$

$\mathrm{Kr}_{2}^{+}$

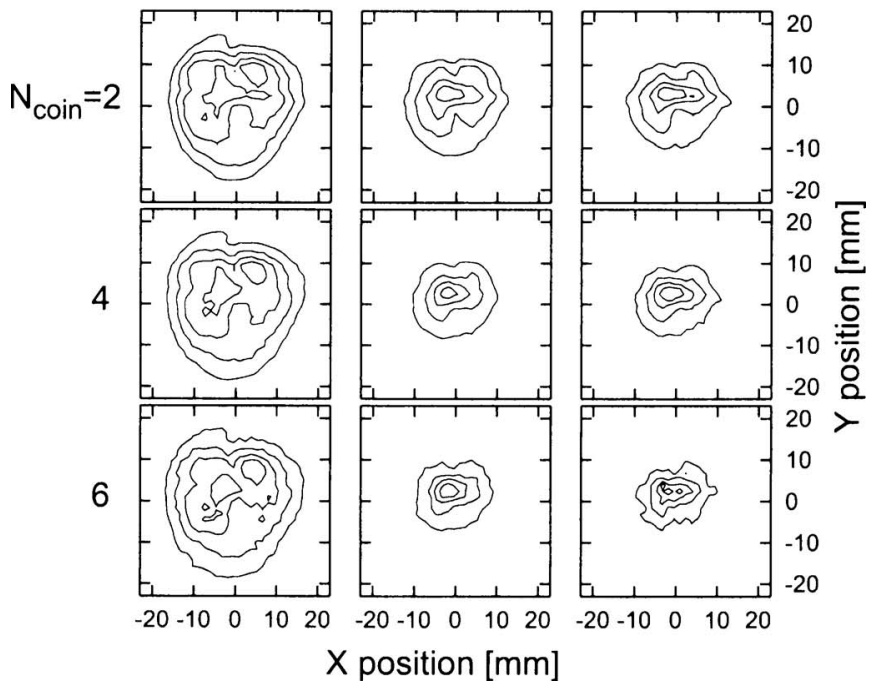

FIG. 6. PSD images of fragment ions from (a) atomic and (b) cluster beams. The contour interval is every $20 \%$ of the maximum count. In (a) all multiply charged ions $\mathrm{Kr}^{z+}(z \geqslant 2)$ are included. In (b) the PSD images are sorted by $N_{\text {coin }}$ and the ion species. In (c) the directions of the x-ray beam and sample beam are drawn (see also Fig. 1).

$\mathrm{Kr}_{3}^{+}$. It should be noted that the distribution of $\mathrm{Kr}_{2}^{+}$as well as that of $\mathrm{Kr}_{3}^{+}$shrink with increasing $N_{\text {coin }}$, whereas that of $\mathrm{Kr}^{+}$ remains very broad. The different $N_{\text {coin }}$ dependence of PSD image between $\mathrm{Kr}^{+}$and $\mathrm{Kr}_{2}^{+}$(or $\mathrm{Kr}_{3}^{+}$) is consistent with that of the TOF peak width shown in Fig. 3(c).

\section{DISCUSSION}

\section{A. Examination of the reliability of our experiment}

Before getting a physical picture from our experimental results, we should examine the reliability of our experiment, because, to our knowledge, no work has been done to detect more than four ions coincidently using the delay-line anode detector. First, we check the multihit capability of ions, because, when two ions reach the detector successively within a dead time of the detector, confusion or missing of signals may occur, leading to false coincidences. Figure 2 shows the time difference between series ions, where false coincidences are removed. This indicates that the effective dead time is about $40 \mathrm{~ns}$ or less. Moreover, since the observed peak width (e.g., $500 \mathrm{~ns}$ for $\mathrm{Kr}^{+}$) in the time-of-flight spectra is by far larger than the dead time (i.e., $40 \mathrm{~ns}$ ), it is not surprising that several $\mathrm{Kr}^{+}$ions were detected in coincidence.

Another unfavorable possibility is that what we observed might be artifacts due to the ringing of the electronic signals 

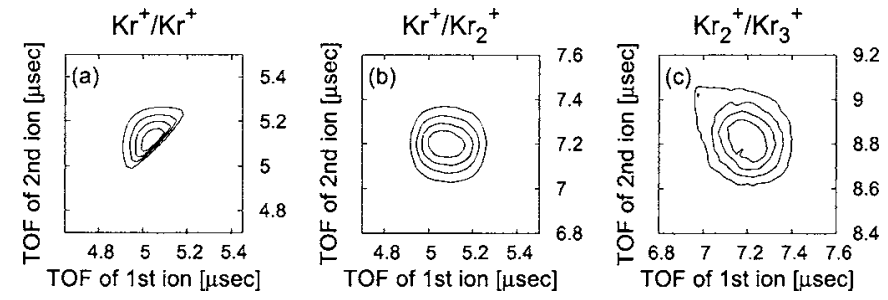

FIG. 7. PIPICO images for $N_{\text {coin }}=2$. The correlations between (a) $\mathrm{Kr}^{+}$and $\mathrm{Kr}^{+}$, (b) $\mathrm{Kr}^{+}$and $\mathrm{Kr}_{2}^{+}$, and (c) $\mathrm{Kr}_{2}^{+}$and $\mathrm{Kr}_{3}^{+}$are displayed.

or so called "ion feedback" in MCPs. In order to check this possibility, two dimensional photoion-photoion coincidence (PIPICO) images, where the TOF of one ion is plotted against the TOF of another ion, are displayed from our multihit data. For the ringing effects, where there exists a relation between the TOF of the $i$ th ion and $j$ th ion, $T(j)$ $=T(i)+(j-i) T_{0}, T_{0}$ being constant, straight lines with slope of 1 would appear in the PIPICO images. In Fig. 7, PIPICO images for $N_{\text {coin }}=2$ are displayed and very similar to those of the previous work on Ar clusters. ${ }^{12}$ Figure 8 represents 1 st and $n$th ion PIPICO images $(n=2,4,6)$ for $N_{\text {coin }}=6$. As shown in Figs. 7 and 8, there are no straight lines with slope of 1 . Therefore, the possibilities of the ringing and ion feedback are definitely excluded.

\section{B. Fragmentation pattern}

It is well known that the Auger decay occurs predominantly within an atom that absorbs an x-ray photon. On the other hand, the Coulomb explosion does not occur without charge separation. Thus one encounters an interesting problem of at what stage of the deexcitation process the interatomic charge separation becomes dominant when a cluster is core excited. Hayakawa et al. proposed a two-step model to interpret the Coulomb explosion pattern of $K$-shell excited selenium dimer. ${ }^{28}$ In this model, the first step is the Auger decay which is the same as the one that occurs in an isolated atom, and the second step is the redistribution of proliferated charges within a dimer. They concluded that the second step starts when the holes reach the $4 p$ valence orbitals, which form the covalent bonds. In what follows, we demonstrate that the two-step model is also applicable to the $\mathrm{Kr}$ clusters to reproduce the number of photodissociation events as a function of $N_{\text {coin }}$.

When a photoionized cluster gets $Z$ positive charges after Auger decay, $Z$ singly charged fragment ions are expected to appear after the Coulomb explosion. In principle all the fragment ions would be detected, but in practice the number
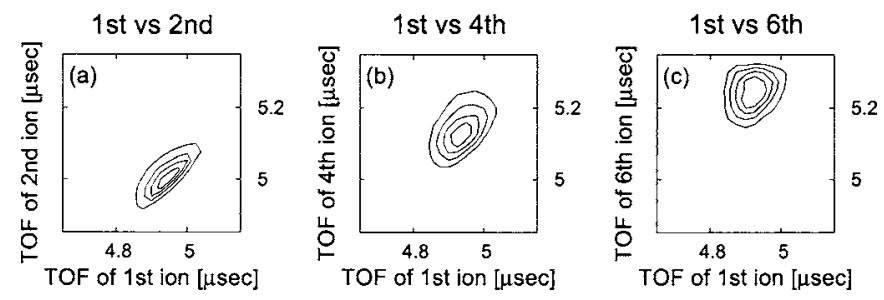

FIG. 8. PIPICO images of $\left(\mathrm{Kr}^{+} / \mathrm{Kr}^{+}\right)$for $N_{\text {coin }}=6$. PIPICO images of (a) The first and second ions, (b) the first and fourth ions, and (c) the first and sixth ions are displayed. of detected ions $N_{\text {coin }}$ is usually less than $Z$ because the detection efficiency $p$ is about $35 \%$. Assuming that the detection of different ions can be treated as an independent event, one can calculate the distribution function of $N_{\text {coin }}, g\left(N_{\text {coin }}\right)$, as follows:

$$
g\left(N_{\text {coin }}\right)=\sum_{Z=N_{\text {coin }}}^{11}{ }_{Z} C_{N_{\text {coin }}} p^{N_{\text {coin }}(1-p)^{Z-N_{\text {coin }}} f(Z) .}
$$

Here $f(Z)$ is a distribution function of $Z$ when the Auger cascade decay is ended. Generally, $f(Z)$ should have a dependence on the parent cluster size $N$, but we assume here that $f(Z)$ is independent of $N$ and hence equal to the relative abundance of $\mathrm{Kr}^{Z+}$ generated from an isolated atom [see Fig. 3(a)]. In spite of such simplification, the calculated $g\left(N_{\text {coin }}\right)$ denoted by a curve in Fig. 5 reproduces the experimental points very well, which supports the validity of the two-step model.

For rare-gas dimers and clusters, interatomic coulombic decay (ICD), ${ }^{29-34}$ in which the energy transfer by a virtual photon exchange can generate a hole in an adjacent atom, was theoretically predicted and experimentally proven recently. If the ICD is considered, the distribution of $Z$ may be shifted to slightly higher $Z$, but it is difficult to evaluate the ICD effects at this stage.

Next, we estimate the average number of charges $\left\langle Z\left(N_{\text {coin }}\right)\right\rangle$ for each $N_{\text {coin }}$ by using

$$
\begin{aligned}
\left\langle Z\left(N_{\text {coin }}\right)\right\rangle= & \frac{1}{g\left(N_{\text {coin }}\right)} \sum_{Z=N_{\text {coin }}}^{11}{ }_{Z} C_{N_{\text {coin }}} \\
& \times p^{N_{\text {coin }}}(1-p)^{Z-N_{\text {coin }} f(Z) Z .}
\end{aligned}
$$

The calculated values of $\left\langle Z\left(N_{\text {coin }}\right)\right\rangle$ are listed in Table I. It is natural that $\left\langle Z\left(N_{\text {coin }}\right)\right\rangle$ increases with $N_{\text {coin }}$ because the number of fragment ions becomes larger with increasing the charge number of parent clusters. Hence, the larger $N_{\text {coin }}$ is, the larger Coulomb energy is stored before explosion.

In conventional coincidence momentum imaging for small molecules, spatial correlations among the fragment ions are displayed graphically by the help of, e.g., Newton diagrams. ${ }^{3,17}$ In the present study for Kr clusters, however, such a graphical representation is not easy because the number of generated charges is too large. Nevertheless, the relative abundance of fragment ions $\mathrm{Kr}_{n}^{+}$shown in Fig. 4 is still meaningful as long as the the detection of different ions can be treated as on independent event. Thus, one can deduce average fragmentation patterns by combining the results in Fig. 4 with those in Table I as follows:

$$
\begin{aligned}
& \mathrm{Kr}_{N}^{6+} \rightarrow 4.2 \mathrm{Kr}^{+}+1.4 \mathrm{Kr}_{2}^{+}+0.4 \mathrm{Kr}_{3}^{+}+\text {(neutral fragments), } \\
& \mathrm{Kr}_{N}^{7+} \rightarrow 5.6 \mathrm{Kr}^{+}+1.1 \mathrm{Kr}_{2}^{+}+0.3 \mathrm{Kr}_{3}^{+}+\text {(neutral fragments), } \\
& \mathrm{Kr}_{N}^{8+} \rightarrow 6.6 \mathrm{Kr}^{+}+1.1 \mathrm{Kr}_{2}^{+}+0.3 \mathrm{Kr}_{3}^{+}+\text {(neutral fragments), } \\
& \mathrm{Kr}_{N}^{9+} \rightarrow 7.4 \mathrm{Kr}^{+}+1.2 \mathrm{Kr}_{2}^{+}+0.4 \mathrm{Kr}_{3}^{+}+\text {(neutral fragments). }
\end{aligned}
$$

It is interesting that the number of $\mathrm{Kr}_{2}^{+}$and $\mathrm{Kr}_{3}^{+}$is almost constant, while that of $\mathrm{Kr}^{+}$increases with the charge number 
TABLE I. Correlation between $N_{\text {coin }}$ events and average charge number of parent cluster ions $\mathrm{Kr}_{N}^{Z+}$.

\begin{tabular}{ccccccccc}
\hline \hline$N_{\text {coin }}$ & 1 & 2 & 3 & 4 & 5 & 6 & 7 & 8 \\
$\left\langle Z\left(N_{\text {coin }}\right)\right\rangle$ & 5.4 & 6.0 & 6.7 & 7.4 & 8.1 & 8.7 & 9.2 & 9.6 \\
\hline \hline
\end{tabular}

of the parent cluster. This feature is different from the observation for triply charged Ar clusters induced by $2 p$ excitation, where $\mathrm{Ar}^{+}$is the most abundant for small clusters and $\mathrm{Ar}_{2}^{+}$becomes more important as the cluster size increases. ${ }^{9,15}$

\section{Momentum distribution}

The momentum vector $\mathbf{p}=\left(p_{x}, p_{y}, p_{z}\right)$ of the fragment ions immediately after the Coulomb explosion can be derived from the detected position on the PSD, $(X, Y)$, and the time-of-flight $T$, by using the following well-known equations: ${ }^{35}$

$$
\begin{aligned}
& p_{x}=\frac{m\left(X-X_{0}\right)}{T}, \quad p_{y}=\frac{m\left(Y-Y_{0}\right)}{T}, \\
& p_{z}=q \frac{U_{G}-U_{R}}{d}\left(T_{0}-T\right),
\end{aligned}
$$

where $m$ and $q$ are the mass and charge of the ion, respectively, $\left(X_{0}, Y_{0}\right)$ is the position on the PSD where an ion with $p_{x}=p_{y}=0$ would be detected, $T_{0}$ is the TOF of a fragment ion with $p_{z}=0, d$ is the spacing between the ground and repeller electrodes, and $U$ is the voltage applied to each electrode (see Fig. 1).

Momentum resolutions are expressed as $\Delta p_{x} \sim m \Delta x / T$, $\Delta p_{y} \sim m \Delta y / T$, and $\Delta p_{z} \sim q\left(U_{G}-U_{\text {rep }}\right) \Delta T / d$, where $\Delta x$ and $\Delta y$ are spatial resolutions estimated from the widths of the PSD image for the atomic beam [see Fig. 6(a)] and $\Delta T$ is a time resolution. Using the relative time resolution $T / \Delta T$ $=220$, we have estimated $\Delta T$ of $\mathrm{Kr}^{+}$and $\mathrm{Kr}_{2}^{+}$to be 23 and $33 \mathrm{~ns}$, respectively. Thus the momentum resolutions are estimated to be $\Delta p_{x} \sim 104 \times 10^{3} \mathrm{amu} / \mathrm{s}, \quad \Delta p_{y} \sim 38$ $\times 10^{3} \mathrm{amu} \mathrm{m} / \mathrm{s}$, and $\Delta p_{z} \sim 44 \times 10^{3} \mathrm{amu} \mathrm{m} / \mathrm{s}$ for $\mathrm{Kr}^{+}$, and $\Delta p_{x} \sim 146 \times 10^{3} \mathrm{amu} \mathrm{m} / \mathrm{s}, \quad \Delta p_{y} \sim 53 \times 10^{3} \mathrm{amu} \mathrm{m} / \mathrm{s}, \quad$ and $\Delta p_{z} \sim 62 \times 10^{3} \mathrm{amu} \mathrm{m} / \mathrm{s}$ for $\mathrm{Kr}_{2}^{+}$. In what follows, we deal only with the magnitude of momentum, $p=\sqrt{p_{x}^{2}+p_{y}^{2}+p_{z}^{2}}$. In Fig. 9 the momentum distributions of $\mathrm{Kr}^{+}$and $\mathrm{Kr}_{2}^{+}$ions are displayed for various $N_{\text {coin. }}$. Although the distributions are rather broad, for example, the peak width of $\mathrm{Kr}^{+}$is $208 \times 10^{3} \mathrm{amu} \mathrm{m} / \mathrm{s}$ for $N_{\text {coin }}=2$, they depend clearly on $N_{\text {coin }}$. The average momentum of $\mathrm{Kr}^{+}$increases moderately with $N_{\text {coin }}$, while that of $\mathrm{Kr}_{2}^{+}$(and also $\mathrm{Kr}_{3}^{+}$, not shown) decreases definitely. Since the total Coulomb energy, which supplies the kinetic energy to the fragment ions, increases with the number of generated charges or $N_{\text {coin }}$ (see Table I), the above results for dimer (and also for trimer) ions look contradictory. However, such contradiction can be resolved, if we assume that the heavier ions are produced in the central part of clusters where the Coulomb interactions from the surrounding ions are more effectively canceled out due to the higher symmetry. More precise discussion will be given in the Appendix.

The assumption mentioned above is consistent with our recent EICO results, ${ }^{23}$ from which we have suggested that the multiply charged monomer ions derive from the cluster surface, the singly charged monomer ions from interior of cluster, and the singly-charged dimer and trimer ions from the core of cluster. It is expected that these heavier ions behaved as a chromophore ionic core ${ }^{36}$ which have a linear conformation because of the directional character of the empty $p$ orbital. ${ }^{37}$

Finally, we estimate the kinetic energy release (KER) from the multiply charged clusters. For example, the fragmentation pattern of $\mathrm{Kr}_{N}^{6+}$ shows that there appear $4.2 \mathrm{Kr}^{+}$, $1.4 \mathrm{Kr}_{2}^{+}$, and $0.4 \mathrm{Kr}_{2}^{+}$on average. From the momentum distribution of $\mathrm{Kr}^{+}, \mathrm{Kr}_{2}^{+}$, and $\mathrm{Kr}_{3}^{+}$for $N_{\text {coin }}=2$, the average kinetic energies of $\mathrm{Kr}^{+}, \mathrm{Kr}_{2}^{+}$, and $\mathrm{Kr}_{3}^{+}$are $4.2,2.6$, and $2.5 \mathrm{eV}$, respectively. Thus the average KER of $\operatorname{Kr}_{N}^{6+}(\langle N\rangle \sim 160)$ is estimated to be $22 \mathrm{eV}$. Using this KER value, one may discuss how far the generated holes are extended within the cluster before the Coulomb explosion. If we assume that the KER is equal to the Coulomb energy stored in the cluster charged with $+6 e$, then the average distance between the center and surrounding ions is estimated to be $\sim 8 \AA$, which corresponds to the second neighbor distance between atoms. Here we assume that the charge separation takes place isotropically. If we take account of the kinetic energy of neutral fragments ${ }^{38}$ which results from collisions with ions during the Coulomb explosion, smaller charge separation distance is (a) $\mathrm{Kr}^{+}$

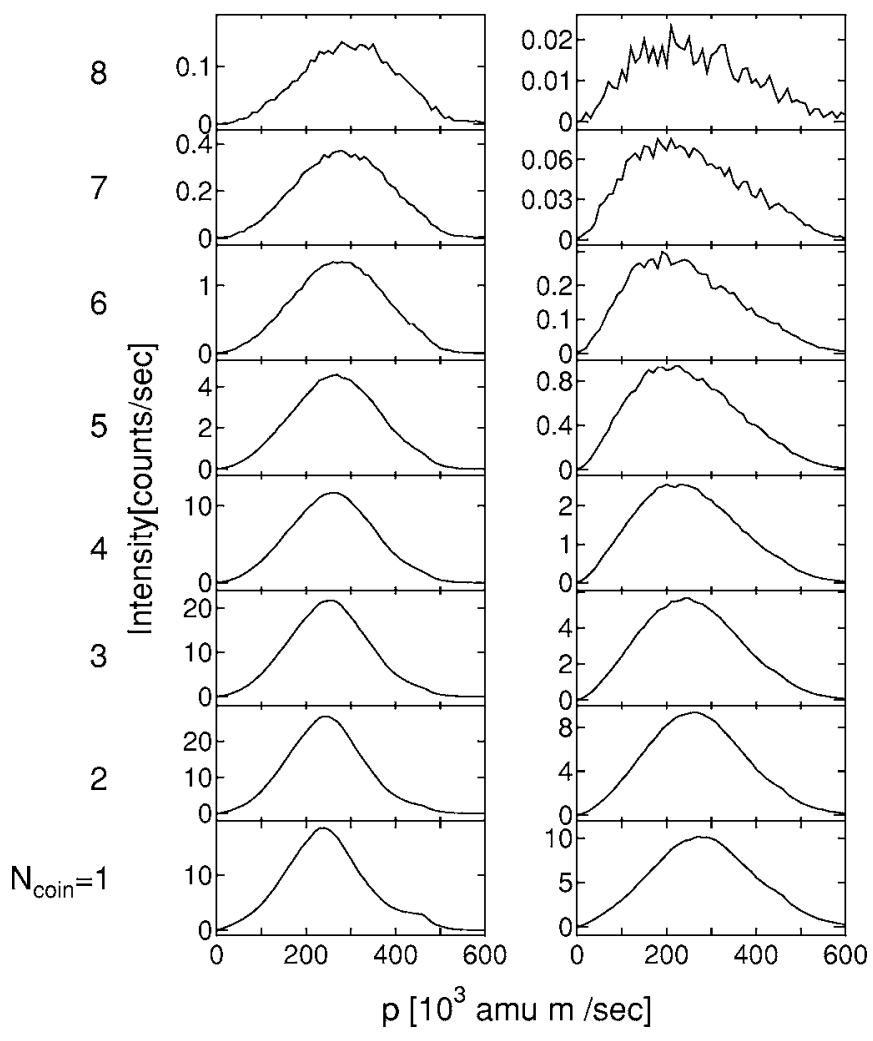

FIG. 9. Momentum distributions of (a) $\mathrm{Kr}^{+}$and (b) $\mathrm{Kr}_{2}^{+}$for each $N_{\text {coin }}$. 
obtained. Therefore, the value of $8 \AA$ estimated only from the charged fragments gives an upper limit of the charge separation distance. The result clearly indicates that the charges in the $\mathrm{Kr}$ clusters are fairly localized in accordance with insulating properties of rare-gas elements.

\section{CONCLUSION}

The electron- $n$-ion coincidence TOF spectra with $n \leqslant 8$ were measured for a $\mathrm{Kr}$ cluster beam $(\langle N\rangle \sim 160)$ together with the momentum distributions of ions by utilizing hard $\mathrm{x}$-ray. We have sorted the TOF spectra by the number of coincidence ion signals $N_{\text {coin }}$ and found that singly charged fragment ions such as $\mathrm{Kr}^{+}, \mathrm{Kr}_{2}^{+}$, and $\mathrm{Kr}_{3}^{+}$are dominant for $N_{\text {coin }} \geqslant 2$, while multiply charged ions are detected mainly for $N_{\text {coin }}=1$. This indicates a fast charge separation process before the Coulomb explosion. The observed $N_{\text {coin }}$ dependence of the number of photodissociation events can be explained by a two-step model where the first step is the Auger decay which is the same as the one that occurs in an isolated atom, and the second step is the redistribution of proliferated charges within the cluster. The $N_{\text {coin }}$ dependence of the peak widths in the TOF spectra reveals that the average momentum of the $\mathrm{Kr}^{+}$ions increases with $N_{\text {coin }}$, while those of $\mathrm{Kr}_{2}^{+}$ and $\mathrm{Kr}_{3}^{+}$decrease. These results have been more directly confirmed by the momentum imaging measurements. Based upon these experimental observations, we have concluded that the heavier ions are produced in the central part of clusters where the Coulomb interactions from the surrounding ions are more effectively canceled out due to the higher symmetry. This gives a strong support for the site-dependent decay processes suggested by our group ${ }^{23}$ and for the existence of chromophore core that has been evidenced mainly by optical properties. ${ }^{36,37}$

\section{ACKNOWLEDGMENTS}

The experiments described in this paper were done as a proposal 2005B0751 of SPring-8. This work was supported by the Grant-in-Aid for Scientific Research from the JPSJ (Nos. 12554013, 15651044, 16201021, and 18710087) and the Grant-in-Aid for the 21st Century COE "Center for Diversity and Universality in Physics" from the MEXT of Japan.

\section{APPENDIX: ION MOMENTUM AFTER COULOMB EXPLOSION}

Let us interpret the assumption made in Sec. IV B by the help of equations. The momentum before the Coulomb explosion is regarded as small compared with that after the explosion, while the Coulomb energy after the explosion is negligibly small compared with that before the explosion. Indeed, this is a basis for the notion of kinetic energy release, especially when a center-of-mass system is adopted. Thus the momentum of the $j$ th ion immediately after the explosion may be written by a differential equation as

$$
\mathbf{p}_{j} \cong \tau_{j} \sum_{i \neq j}^{Z} \mathbf{f}\left(\mathbf{r}_{i}, \mathbf{r}_{j}\right),
$$

where

$$
\mathbf{f}\left(\mathbf{r}_{i}, \mathbf{r}_{j}\right)=\frac{e^{2}\left(\mathbf{r}_{i}-\mathbf{r}_{j}\right)}{\left|\mathbf{r}_{i}-\mathbf{r}_{j}\right|^{3}}
$$

is the Coulomb force acting on the $j$ th ion located at $\mathbf{r}_{j}$ from the $i$ th ion at $\mathbf{r}_{i}$, and $\tau_{j}$ is a very short time during which the Coulomb forces are effectively operating. Here van der Waals force between neutral atoms is ignored. From these equations one can readily see that the sum of the Coulomb forces could be practically canceled out when the $j$ th ion is surrounded by other ions isotropically. Especially, $\mathbf{p}_{j}$ is rigorously null, when the $j$ th ion is located at the center of gravity and at the same distance from other ions, and these ions have the same mass.

Here we have neglected influences of neutral atoms on the $j$ th ion. It is indeed conceivable that the collision between the ion and neutrals causes the reduction of the ionic momentum. However, at least as for $\mathrm{Kr}_{2}^{+}$ions, the influences are not likely to be significant, because their average momentum decreases with increasing $Z\left(N_{\text {coin }}\right)$, that is, with a slight decrease of the number of neutrals [see Fig. 9(b)].

${ }^{1}$ J. Purnell, E. M. Snyder, S. Wei, and A. W. Castleman, Chem. Phys. Lett. 229, 333 (1994).

${ }^{2}$ M. Hirokane, S. Shimizu, M. Hashida, S. Okada, S. Okihara, F. Sato, T. Iida, and S. Sakabe, Phys. Rev. A 69, 063201 (2004).

${ }^{3}$ H. Hasegawa, A. Hishikawa, and K. Yamanouchi, Chem. Phys. Lett. 349, 57 (2001).

${ }^{4}$ H. Wabnitz, L. Bittner, A. R. B. de Castro et al., Nature (London) 420, 482 (2002).

${ }^{5}$ W. Tappe, R. Flesch, E. Rühl, R. Hoekstra, and T. Schlathölter, Phys. Rev. Lett. 88, 143401 (2002)

${ }^{6}$ S. Martin, L. Chen, A. Denis, and J. Désesquelles, Phys. Rev. A 59, R1734 (1999).

${ }^{7}$ T. A. Carlson, Photoelectron and Auger Spectroscopy (Plenum, New York, 1975).

${ }^{8}$ T. A. Carlson, W. E. Hunt, and M. O. Krause, Phys. Rev. 151, 41 (1966).

${ }^{9}$ E. Rühl, Int. J. Mass. Spectrom. 229, 117 (2003).

${ }^{10}$ R. Kambach, M. Joppien, J. Stapelfeldt, J. Wörmer, and T. Möller, Rev. Sci. Instrum. 64, 2838 (1993).

${ }^{11}$ E. Rühl, C. Schmale, H. W. Jochims, E. Biller, M. Simon, and H. Baumgärtel, J. Chem. Phys. 95, 6544 (1991).

${ }^{12}$ E. Rühl, C. Heinzel, H. Baumgärtel, M. Lavollée, and P. Morin, Z. Phys. D: At., Mol. Clusters 31, 245 (1994).

${ }^{13}$ E. Rühl, C. Heinzel, A. P. Hitchcock, and H. Baumgärtel, J. Chem. Phys. 98, 2653 (1993).

${ }^{14}$ M. Gisselbrecht, A. Lindgren, M. Tchaplyguine et al., J. Chem. Phys. 123, 194301 (2005).

${ }^{15}$ E. Rühl, A. Knop, A. P. Hitchcock, P. A. Dowben, and D. N. Mcilroy, Surf. Rev. Lett. 3, 557 (1996).

${ }^{16}$ A. de Fanis, M. Oura, N. Saito et al., J. Phys. B 37, L235 (2004).

${ }^{17}$ K. Ueda and J. H. D. Eland, J. Phys. B 38, S839 (2005).

${ }^{18}$ E. Rühl, C. Heinzel, A. P. Hitchcock, H. Schmelz, C. Reynaud, H. Baumgärtel, W. Drube, and R. Frahm, J. Chem. Phys. 98, 6820 (1993).

${ }^{19}$ K. Nagaya, M. Yao, T. Hayakawa, Y. Ohmasa, Y. Kajihara, M. Ishii, and Y. Katayama, Phys. Rev. Lett. 89, 243401 (2002).

${ }^{20}$ A. Knop, B. Wassermann, and E. Rühl, Phys. Rev. Lett. 80, 2302 (1998).

${ }^{21}$ T. Hatsui, H. Setoyama, N. Kosugi, B. Wassermann, I. L. Bradeanu, and E. Rühl, J. Chem. Phys. 123, 154304 (2005).

${ }^{22}$ M. Lengen, M. Joppien, R. Müller, J. Wörmer, and T. Möller, Phys. Rev. Lett. 68, 2362 (1992).

${ }^{23}$ K. Nagaya, M. Yao, H. Murakami, A. Mori, Y. Ohmasa, and H. Kajikawa, J. Phys. Soc. Jpn. 75, 114801 (2006).

${ }^{24}$ O. F. Hagena, Rev. Sci. Instrum. 63, 2374 (1992). 
${ }^{25}$ W. C. Wiley and I. H. McLaren, Rev. Sci. Instrum. 26, 1150 (1955).

${ }^{26}$ M. Lezius, P. Scheier, A. Stamatovic, and T. D. Märk, J. Chem. Phys. 91, 3240 (1989).

${ }^{27}$ T. D. Märk, Int. J. Mass Spectrom. Ion Process. 79, 1 (1987).

${ }^{28}$ T. Hayakawa, K. Nagaya, I. Yamamoto, Y. Ohmasa, M. Yao, and M. Nomura, J. Phys. Soc. Jpn. 69, 2039 (2000).

${ }^{29}$ R. Santra and L. S. Cederbaum, Phys. Rev. Lett. 90, 153401 (2003).

${ }^{30}$ J. Zobeley, R. Santra, and L. S. Cederbaum, J. Chem. Phys. 115, 5076 (2001).

${ }^{31}$ G. Öhrwall, M. Tchaplyguine, M. Lundwall et al., Phys. Rev. Lett. 93, 173401 (2004).

${ }^{32}$ S. Marburger, O. Kugeler, U. Hergenhan, and T. Möller, Phys. Rev. Lett. 90, 203401 (2003).
${ }^{33}$ T. Jahnke, A. Czasch, M. S. Schöffler et al., Phys. Rev. Lett. 93, 163401 (2004).

${ }^{34}$ Y. Morishita, X.-J. Liu, T. Lischke, M. Kato, G. Prümper, M. Oura, H. Yamaoka, Y. Tamenori, I. H. Suzuki, and K. Ueda, Phys. Rev. Lett. 96, 243402 (2006).

${ }^{35}$ R. Dörner, V. Mergel, O. Jagutzki, L. Spielberger, J. Ullrich, R. Moshammer, and H. Schmidt-Böcking, Phys. Rep. 330, 95 (2000).

${ }^{36}$ N. E. Levinger, D. Ray, M. L. Alexander, and W. C. Lineberger, J. Chem. Phys. 89, 5654 (1988).

${ }^{37}$ H. Haberland, B. von Issendorff, T. Kolar, H. Kornmeier, C. Ludewigt, and A. Risch, Phys. Rev. Lett. 67, 3290 (1991).

${ }^{38}$ A. B. Jones, P. R. Jukes, and A. J. Stace, J. Chem. Phys. 111, 959 (1999). 